POS $\quad$ PROCEEDINGS

\title{
Lattice Predictions for Bound Heavy Tetraquarks
}

\section{Anthony Francis*}

Theoretical Physics Department, CERN, CH-1211 Geneva 23, Switzerland

E-mail: anthony. francisecern.ch

\section{Renwick J. Hudspith}

Department of Physics and Astronomy, York University, Toronto, Ontario, M3J 1P3, Canada

E-mail: renwick.james.hudspith@googlemail.com

\section{Randy Lewis}

Department of Physics and Astronomy, York University, Toronto, Ontario, M3J 1P3, Canada

E-mail: randy. lewis@yorku.ca

\section{Kim Maltman}

Department of Mathematics \& Statistics, York University, Toronto, ON M3J 1P3, Canada CSSM, University of Adelaide, Adelaide SA 5005, Australia

E-mail: kmaltman@yorku.ca

We investigate the possibility of doubly heavy $q q^{\prime} \bar{Q} \bar{Q}^{\prime}$ tetraquark bound states using $n_{f}=2+1$ lattice QCD with pion masses $\simeq 164,299$ and $415 \mathrm{MeV}$. Two types of lattice interpolating operator are chosen, reflecting first diquark-antidiquark and second meson-meson structure. Performing variational analyses using these operators and their mixings, we determine the ground and first excited states from the lattice correlators. Using non-relativistic QCD to simulate the bottom quarks and the Tsukuba formulation of relativistic heavy quarks for charm quarks, we study the $u d \bar{b} \bar{b}, \ell s \bar{b} \bar{b}$ as well as $u d \bar{c} \bar{b}$, channels with $\ell=u, d$. In the case of the $u d \bar{b} \bar{b}$ and $\ell s \bar{b} \bar{b}$ channels unambiguous signals for $J^{P}=1^{+}$tetraquarks are found with binding energies 189(10) and 98(7) $\mathrm{MeV}$ below the corresponding free two-meson thresholds at the physical point. These tetraquarks are therefore not only strong-interaction, but also electromagnetic-interaction stable, and can decay only weakly. So far these are the first exotic hadrons predicted to have this feature. Further evidence for binding is found in the $u d \bar{c} \bar{b}$ channel, where the binding energy broadly straddles the electromagnetic stability threshold. We also study the dependence of the tetraquark binding on heavy quark mass by considering the channels $u d \bar{b}^{\prime} \bar{b}^{\prime}, \ell s \bar{b}^{\prime} \bar{b}^{\prime}$ as well as $u d \bar{b}^{\prime} \bar{b}, \ell s \bar{b}^{\prime} \bar{b}$ involving a heavy $b^{\prime}$ quark with mass between roughly 0.6 and 6.3 times the physical $b$ quark mass. The observed mass dependence of these four flavor channels is shown to follow closely a phenomenological form expected on simple physical grounds.

The 39th International Conference on High Energy Physics (ICHEP2018)

4-11 July, 2018

Seoul, South Korea

\footnotetext{
* Speaker.
} 


\section{A binding mechanism for heavy-light tetraquarks}

The phenomenological concepts of good diquark attraction and heavy quark spin symmetry suggest a binding mechanism to generate stable $J^{P}=1^{+}$tetraquarks made up of two light quarks and two heavy anti-quarks, see [1, 2,3] and [4] for a review. Considering $u d \bar{b}^{\prime} \bar{b}^{\prime}, u d \bar{b}^{\prime} \bar{b}, \ell s \bar{b}^{\prime} \bar{b}^{\prime}$ and $\ell s \bar{b}^{\prime} \bar{b}$ flavor combinations this notion can be further quantified in four model ansaetze describing the heavy quark mass dependence of their binding energies $\Delta E=E_{\text {tetra }}-E_{\text {thresh. }}$. Defining $r=m_{b} / m_{b^{\prime}}$, we find:

$$
\begin{aligned}
\Delta E_{u d \bar{b}^{\prime} \bar{b}^{\prime}, \ell s \bar{b}^{\prime} \bar{b}^{\prime}} & =\frac{C_{0}}{2 r}+C_{1}^{u d, \ell s}+C_{2}^{u d, \ell s}(2 r)+\left(C_{\bar{b}^{\prime} \bar{b}^{\prime}}^{u d, \ell s}\right) r, \\
\Delta E_{u d \bar{b}^{\prime} \bar{b}, \ell s \bar{b}^{\prime} \bar{b}} & =\frac{C_{0}}{1+r}+C_{1}^{u d, \ell s}+C_{2}^{u d, \ell s}(1+r)+\left(C_{\bar{b}^{\prime} \bar{b}}^{u d, \ell s}(r)\right) \text { for } r<1, \\
\Delta E_{u d \bar{b}^{\prime} \bar{b}, \ell s \bar{b}^{\prime} \bar{b}}= & \frac{C_{0}}{1+r}+C_{1}^{u d, \ell s}+C_{2}^{u d, \ell s}(1+r)+\left(C_{\bar{b}^{\prime} \bar{b}}^{u d, \ell s}(r)\right) \text { for } r>1,
\end{aligned}
$$

where the first terms parametrize color Coulomb attraction between the two heavy anti-quarks and good light diquark attraction, while $C_{\bar{b}^{\prime} \bar{b}^{\prime}, \bar{b}^{\prime} \bar{b}^{\prime}}^{u d}(r)$ encapsulates threshold contributions and is fixed by the observed meson spectrum.

In the following we test the effectiveness of the binding mechanism underlying these phenomenological forms using first principles lattice QCD calculations. We find the forms above describe the heavy quark mass dependence of both $\{b b\}$ and $\left\{b^{\prime} b\right\}$ channels well. In addition, direct calculations provide clear evidence of strong-interaction stable, bound $u d \bar{b} \bar{b}$ and $\ell s \bar{b} \bar{b}$ tetraquarks, as well as first evidence for binding also in the $u d \bar{c} \bar{b}$ channel.

\section{First principles results using lattice QCD}

The tetraquark and two meson threshold energies can be determined from current-current correlation functions in Euclidean time calculated using lattice QCD. We focus on a combination of correlators giving access to the binding energy directly, $G_{\mathscr{O}_{1} \mathscr{O}_{2}}=C_{\mathscr{O}_{1} \mathscr{O}_{2}}(t) / C_{P P}(t) C_{V V}(t)$. To identify the ground state cleanly we solve a generalized eigenvalue problem, GEVP, and monitor the exponential behavior of the lowest lying eigenvalue, $F(t) v=\lambda(t) F\left(t_{0}\right) v \Rightarrow \lambda_{i}(t)=A e^{-\Delta E_{i}\left(t-t_{0}\right)}$, where the correlation matrix $F(t)$ is made up of hadron correlators derived from a chosen basis of interpolating operators. With the phenomenological picture in mind we choose local operators with diquark-antidiquark and meson-meson structures, leading to $2 \times 2$ and a $3 \times 3$ GEVPs for the $\{b b\}$ for the $\left\{b^{\prime} b\right\},\{c b\}$ cases, respectively.

Our calculations are performed on three gauge ensembles with $n_{f}=2+1$ dynamical quark flavors at fixed lattice spacing, $a^{-1}=2.194 \mathrm{GeV}$, with pion masses $m_{\pi}=415,299$ and $164 \mathrm{MeV}$. The configurations were generated by the PACS-CS collaboration [5]. To calculate the hadron correlations functions we use gauge-fixed wall sources for all valence quark flavors. A relativistic heavy quark effective action with non-perturbatively tuned parameters is used for the charm and lattice NRQCD for the bottom quark [3, 6].

\subsection{Bound $u d \bar{b} \bar{b}$ and $\ell s \bar{b} \bar{b}$ tetraquarks}

In [3] we performed the first direct lattice calculation of the binding energies $\Delta E$ for the $u d \bar{b} \bar{b}$ and $\ell s \bar{b} \bar{b}$ tetraquark candidates, as they are the most promising channels for binding given the 

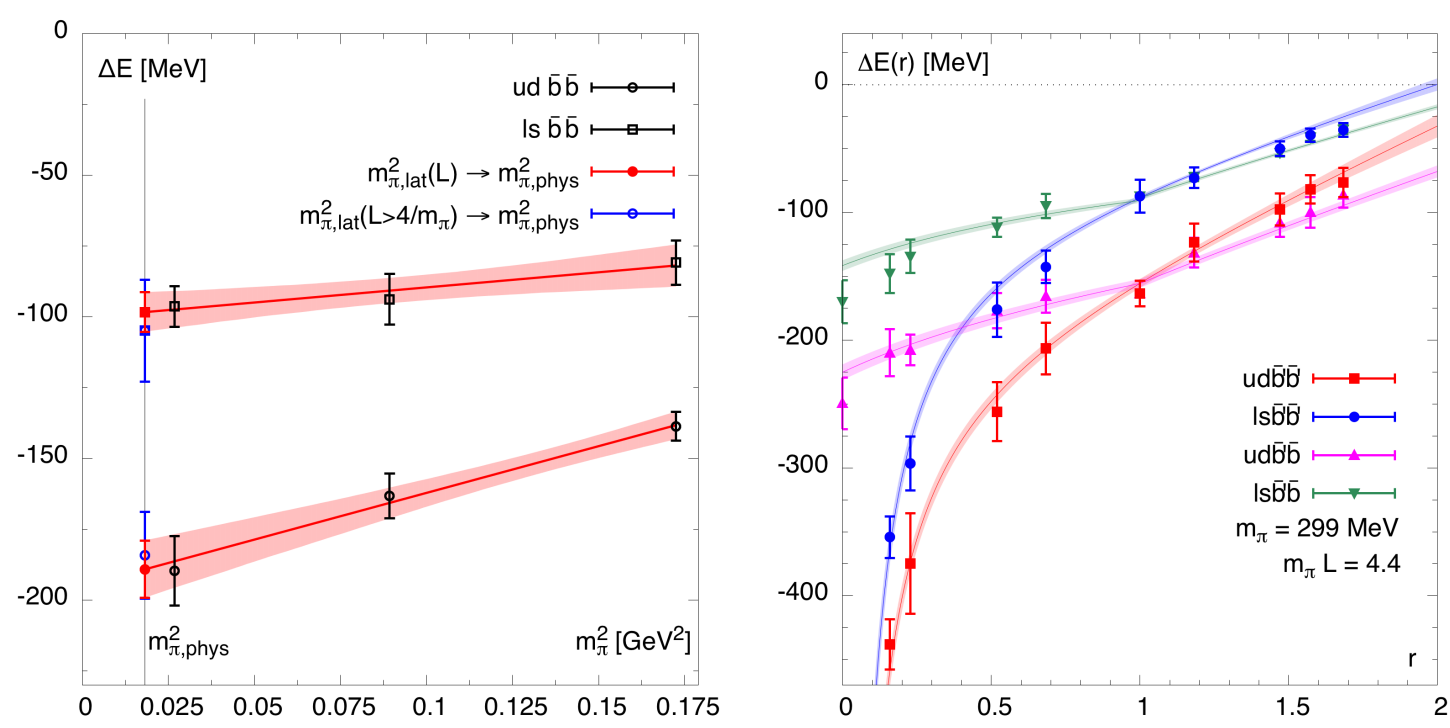

Figure 1: Left: Chiral extrapolations of the $u d \bar{b} \bar{b}$ and $\ell s \bar{b} \bar{b}$ binding energies. Red lines and points show the extrapolations using all three ensembles, the blue points those using $m_{\pi}=415 \mathrm{MeV}$ and $m_{\pi}=299 \mathrm{MeV}$. Right: The dependence on the heavy-quark mass ratio, $r=m_{\text {bare }}^{b} / m_{\text {bare }}^{b^{\prime}}$, of the binding energies for the $u d \bar{b}^{\prime} \bar{b}$, $u d \bar{b}^{\prime} \bar{b}^{\prime}, \ell s b^{\prime} b$ and $\ell s b^{\prime} b^{\prime}$ channels. The solid lines show the fits obtained using Eqs. (1.1)-(1.3).

phenomenological intuition outlined above. The finite volume binding energies are determined by fitting the exponential behavior of the ground state solutions to the above GEVPs, see [3] for more details. They are shown for all three pion masses and both channels in Fig. 1 (left). The results are extrapolated to physical pion mass, once using all ensembles (red) and once cutting the result with $m_{\pi} L<4$ (blue). We find clear evidence for strong-interaction stable tetraquarks with binding energies $\Delta E_{u d \bar{b} \bar{b}}=-189(10)(3) \mathrm{MeV}$ and $\Delta E_{\ell s \bar{b} \bar{b}}=-98(7)(3) \mathrm{MeV}$. This implies both states can decay only weakly, a unique feature for these tetraquarks.

\subsection{Heavy quark mass dependence of heavy-light tetraquarks}

To further increase our understanding of the underlying binding mechanism we study the heavy quark mass dependence of the four $J^{P}=1^{+}$tetraquark channels $u d \bar{b}^{\prime} \bar{b}^{\prime} u d \bar{b}^{\prime} \bar{b}$, $\ell s \bar{b}^{\prime} \bar{b}^{\prime}$ and $\ell s \bar{b}^{\prime} \bar{b}$ using a variable unphysical heavy quark $b^{\prime}$ with $m_{b^{\prime}} / m_{b} \sim 6.29,4.40,1.93,1.46,0.85,0.68,0.64$ and 0.60. This investigation also allows us to infer which channels (if any) are likely to support a bound state as $m_{b^{\prime}} \rightarrow m_{c}$, where a direct lattice calculation is significantly more expensive. The resulting binding energies, shown in Fig. 1 (right), are determined similarly to above (see [6, 7]). Fitting the phenomenological model ansaetze, Eqs. (1.1)-(1.3), we achieve a good description of the data. This is an encouraging validation of phenomenological intuition and hints that the most likely bound tetraquark candidate with one or both of the heavy quarks being charm is the $u d \bar{c} \bar{b}$.

\subsection{Indications of a bound $u d \bar{c} \bar{b}$ tetraquark}

With the phenomenological study of the previous section identifying the $u d \bar{c} \bar{b}$ channel as the most likely to support a strong interaction stable $J^{P}=1^{+}$tetraquark with non-zero charm, we focus our resources on a direct calculation in this channel. In Fig. 2 we show our results for the 

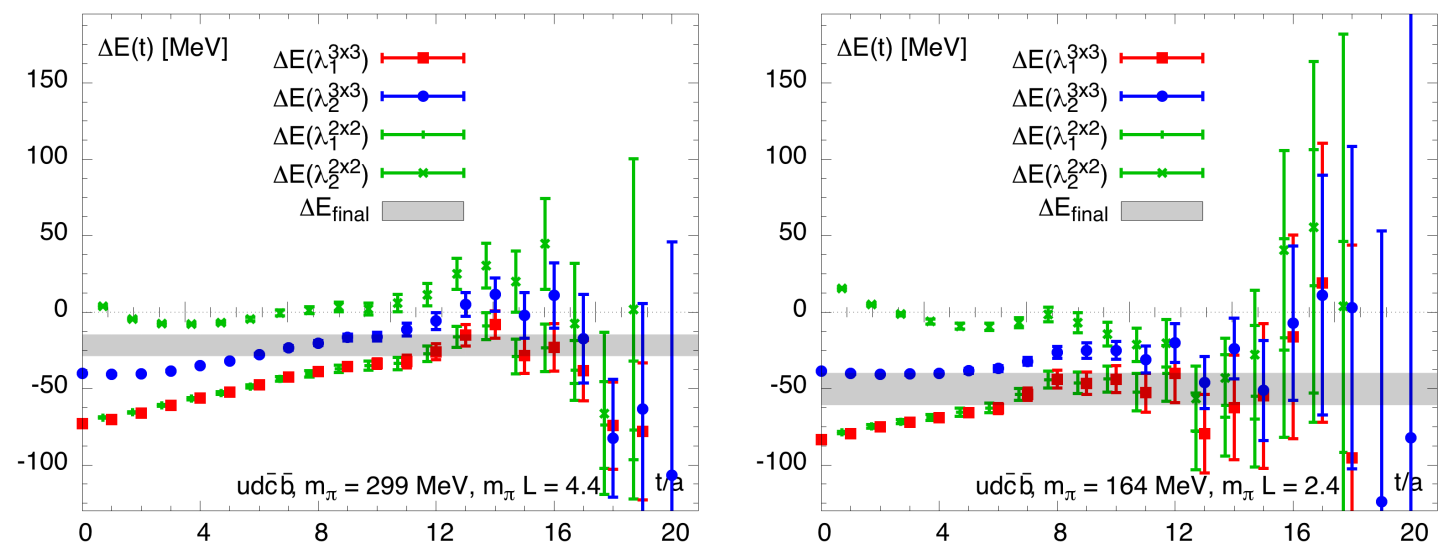

Figure 2: $u d \bar{c} \bar{b}$ tetraquark results for binding energies at $m_{\pi}=299 \mathrm{MeV}$ (left) and $m_{\pi}=164 \mathrm{MeV}$ (right). Red squares and blue circles denote $3 \times 3$ GEVP ground and first excited state results, respectively, green vertical dashes and green diagonal crosses ground and first excited state $2 \times 2$ GEVP results. The grey bands depict the final binding energies, derived from single-exponential fits to the first eigenvalues. Further details may be found in [7], $2 \times 2$ GEVP results are offset slightly in $t$.

effective binding energies on the ensembles with pion masses $m_{\pi}=299 \mathrm{MeV}$ (left) and $164 \mathrm{MeV}$ (right). The first two finite volume state binding energies for both the $2 \times 2$ (green, offset in $t / a$ ) and $3 \times 3$ (colors) GEVPs are given. We fit the correlators obtained from the GEVPs (not shown here) to single exponentials. An in-depth discussion is given in [7]. For both lattice setups we observe ground state energies below the two-meson threshold, thereby providing evidence of the existence of a $u d \bar{c} \bar{b}$ tetraquark. Taking the upper bound of the $m_{\pi}=299 \mathrm{MeV}$ and lower bound of the $m_{\pi}=164 \mathrm{MeV}$ results to provide an estimate for the $u d \bar{c} \bar{b}$ binding energy we find $-61 \mathrm{MeV}<$ $\Delta E_{u d \bar{c} \bar{b}}<-15 \mathrm{MeV}$. Such a charmed-bottom tetraquark should be easier to detect experimentally than the more deeply bound doubly bottom tetraquarks. The dominant systematic uncertainty in this calculation are finite volume effects, making a future detailed finite volume study desirable.

\section{References}

[1] M. Karliner and J. L. Rosner, Phys. Rev. Lett. 119, no. 20, 202001 (2017) doi:10.1103/PhysRevLett.119.202001 [arXiv:1707.07666 [hep-ph]].

[2] E. J. Eichten and C. Quigg, Phys. Rev. Lett. 119, no. 20, 202002 (2017) doi:10.1103/PhysRevLett.119.202002 [arXiv:1707.09575 [hep-ph]].

[3] A. Francis, R. J. Hudspith, R. Lewis and K. Maltman, Phys. Rev. Lett. 118, no. 14, 142001 (2017) doi:10.1103/PhysRevLett.118.142001 [arXiv:1607.05214 [hep-lat]].

[4] J. M. Richard, Few Body Syst. 57, no. 12, 1185 (2016) doi:10.1007/s00601-016-1159-0 [arXiv:1606.08593 [hep-ph]].

[5] S. Aoki et al. [PACS-CS Collaboration], Phys. Rev. D 79, 034503 (2009) doi:10.1103/PhysRevD.79.034503 [arXiv:0807.1661 [hep-lat]].

[6] A. Francis, R. J. Hudspith, R. Lewis and K. Maltman, EPJ Web Conf. 175, 05023 (2018) doi:10.1051/epjconf/201817505023 [arXiv:1711.03380 [hep-lat]].

[7] A. Francis, R. J. Hudspith, R. Lewis and K. Maltman, arXiv:1810.10550 [hep-lat]. 